\title{
Development of Film Dosage Forms Containing Miconazole for the Treatment of Oral Candidiasis
}

\author{
Yoshifumi Murata $^{{ }^{*}}$, Takashi Isobe ${ }^{1}$, Kyoko Kofuji $^{1}$, Norihisa Nishida ${ }^{2}$, Ryosei Kamaguchi ${ }^{2}$ \\ ${ }^{1}$ Faculty of Pharmaceutical Science, Hokuriku University, Kanazawa, Japan; ${ }^{2}$ Morishita Jintan Co. Osaka Technocenter, Hirakata, \\ Japan. \\ Email: *y-murata@hokuriku-u.ac.jp
}

Received April $5^{\text {th }}, 2013$; revised May $10^{\text {th }}, 2013$; accepted May 21 $1^{\text {st }}, 2013$

Copyright (C) 2013 Yoshifumi Murata et al. This is an open access article distributed under the Creative Commons Attribution License, which permits unrestricted use, distribution, and reproduction in any medium, provided the original work is properly cited.

\begin{abstract}
Film dosage forms (FDs) containing miconazole (MCZ) for the treatment of oral candidiasis were prepared using water-soluble polysaccharides, and the dissolution profiles of MCZ from the FDs were investigated. In addition, the forms were modified by the addition of a surface active agent to accelerate the drug dissolution rate. Circular films incorporating $\mathrm{MCZ}$ were obtained using each polysaccharide. Most FDs were easy to handle and resistant to tearing. No diffraction peaks were observed in the X-ray diffractograms of FDs. FDs prepared with sodium alginate or pullulan immediately swelled and disintegrated in aqueous medium, whereas MCZ incorporated in the FD gradually dissolved. A marked acceleration in the MCZ dissolution rate was observed when FD was prepared with polysaccharide containing a surfactant. These results confirmed that modified FDs are useful for treating localized conditions in the oral cavity, such as oral candidiasis, and that FDs can simplify the administration of drugs to patients.
\end{abstract}

Keywords: Film Dosage Form; Miconazole; Oral Candidiasis; Natural Polysaccharide; Surface Active Agent

\section{Introduction}

Films are used as a functional dosage form in oral care. Film dosage forms (FDs) adhere to a surface in the presence of a small amount of liquid and the active compound incorporated in the film is released. FDs prepared with water-soluble polymer easily disintegrate upon contact with saliva, allowing the FD to deliver the incorporated drug quickly to the affected part of the oral cavity $[1,2]$. FDs are also useful for patients who have difficulty swallowing regular oral dosage forms [3]. However, the drug incorporated into the FD should be selected carefully as the drug loading capacity of FDs is typically lower than that of oral disintegration tablets. We have reported that algal polysaccharide sodium alginate (ALG) consisting of $\alpha$-L-guluronate and $\beta$-D-mannuronate, and pullulan (PUL) composed of $\alpha$-D-maltotriose, are useful polymers for preparing FDs [4,5]. These natural polysaccharides are safe, and thin films can be formed using simple methods that do not require dissolution in organic solvents.

It has been recently noted that the frequency of intraoral mucous membrane diseases increases with age [6]

${ }^{*}$ Corresponding author.
Oral candidiasis in particular is evident not only in cancer patients receiving chemotherapy, but also in elderly persons with decreased salivation $[7,8]$. Oral candidiasis is a fungal infection caused by Candida strains such as Candida albicans. In general, Candida strains are not harmful to healthy individuals, but can cause infections in persons with compromised immune systems. Miconazole (MCZ) is an antifungal drug used to treat Candida infections [9]. Typically, gel preparations are topically used to directly apply MCZ to the affected area to avoid the systemic action seen after gastrointestinal absorption $[10,11]$. After administering the gel preparation, the patient has to apply the gel evenly in the oral cavity with their tongue. This procedure is tedious for elderly patients and very difficult for caregivers attempting to apply the gel preparation to the patient.

Attempts have been made to develop new dosage forms for treating oral candidiasis. For example, it was reported that a mucoadhesive MCZ buccal tablet was effective in the treatment of oral candidiasis, with only limited systemic absorption of MCZ [12,13]. A gel dosage form containing solid lipid nanoparticles has also been prepared and characterized [14]. In this study, we prepared FDs containing MCZ and natural polysaccha- 
rides using a casting method. It was expected that MCZ would be active following dissolution of the FD in saliva and thus would be useful for the treatment of oral candidiasis. Therefore, the dissolution profiles of MCZ from FDs were investigated in aqueous medium. In addition, the form was modified by the addition of a surface active agent, a surfactant, to accelerate the drug dissolution rate into limited dissolution medium.

\section{Experimental}

\subsection{Materials}

ALG was obtained from Nacalai Tesque Inc. $(300 \mathrm{cps}$, Kyoto, Japan). Mannuronic acid-rich ALG (ALG-M) was supplied by Kibun Food Chemifa Co. (Tokyo, Japan). PUL was supplied by Hayashibara Biochemical Laboratories (Okayama, Japan). Chondroitin sulfate Ctype (CHS) and MCZ were purchased from Wako Pure Chemicals (Osaka, Japan). Six surfactants, polyoxyethylene sorbitan monooleate (Tween 80; TO-10MV), polyoxyethylene sorbitan monostearate (Tween 60; TS10MV), sorbitan stearate (SS-10MV), polyoxyethylene lauryl ether (BL-4.2), polyoxyethylene sorbitan fatty acid ester (TL-10) and diethyl sebacate (DES-SP), were supplied by Nikko Chemicals Co. Ltd. (Tokyo, Japan). All other chemicals were of reagent grade.

\subsection{FD Preparation}

Polysaccharides $(1.5 \%-4 \%, \mathrm{w} / \mathrm{w})$ were dissolved with agitation in deionized water containing a surfactant $(0 \%$ $0.5 \%(\mathrm{w} / \mathrm{v}))$. Ten $\mathrm{mg}$ of $\mathrm{MCZ}$ was added to $10 \mathrm{~g}$ of film base solution, the mixture was thoroughly mixed, then $3.0 \mathrm{~g}$ of each solution was poured into individual plastic Petri dishes (diameter, $54 \mathrm{~mm}$ ). After $24 \mathrm{~h}$ at $37^{\circ} \mathrm{C}$, the circular films formed on each dish were transferred to a desiccator. Film formation was judged to have failed if either a circular film was not obtained, if the film had cracks, or if the film could not be removed from the bottom of the dish. In the present method, $3 \mathrm{mg}$ of MCZ was theoretically incorporated into each film dosage form.

\subsection{Film Thickness and Rheological Properties}

Film thickness was measured at 10 points on each film using a micrometer (CLM1-15QM; Mitutoyo, Kawasaki, Japan) with a set pressure of $0.5 \mathrm{~N}$. Measurements were made on 3 films, and the mean thickness was calculated for each type. The rheological properties of each film were determined using a rheometer (SUN RHEO TEX SD-700\#; Sun Scientific Co., Tokyo, Japan) at room temperature. The film was fixed on a vial (inner diameter, $1.4 \mathrm{~mm}$; outer diameter, $18.8 \mathrm{~mm}$ ) using joining tape (Scotch mending tape; Sumitomo 3M Ltd., Tokyo, Japan), and was probed with a cylindrical adapter (diame- ter, $5.0 \mathrm{~mm}$ ). Stress and strain were measured at the point at which the adapter broke through the film. The tests were performed in triplicate.

\subsection{Solubility of MCZ}

The solubility of MCZ was measured in physiological saline containing a surfactant. MCZ was added to the test solution and shaken at $37^{\circ} \mathrm{C}$ for $24 \mathrm{~h}$, then the suspension was removed using a pre-heated plastic syringe (Terumo Co., Tokyo) at $37^{\circ} \mathrm{C}$ and filtered using a syringe driven filter unit (Millex-HV, pore size: $0.45 \mu \mathrm{m}$, Millipore Co., MA, USA). The solution was diluted with physiological saline and injected onto an HPLC column.

\subsection{Determination of $\mathrm{MCZ}$}

The HPLC system (Hitachi Co., Tokyo) consisted of a pump (L-2130), UV-detector (L-2400), autosampler (L-2200) and chromate-integrator (D-2500) connected to a packed column $\left(150 \mathrm{~mm} \times 4.6 \mathrm{~mm}\right.$, Cosmosil $5 \mathrm{C}_{18^{-}}$ MS-II, Nacalai Tesque, Kyoto). To determine the concentration of MCZ, HPLC was conducted at ambient temperature using an eluent consisting of $10 \mathrm{mM}$ $\mathrm{KH}_{2} \mathrm{PO}_{4}$ and acetonitrile (1:4) at a flow rate of 0.8 $\mathrm{ml} / \mathrm{min}$ [15]. The detector wavelength was set at $230 \mathrm{~nm}$.

\subsection{X-Ray Diffractometry}

X-ray diffractometry was carried out using an automatic diffractometer (D8 DISCOVER with GADDS; Bruker AXS K.K., Yokohama, Japan) at a voltage of $40 \mathrm{kV}$ and a current of $40 \mathrm{~mA}$. The results of X-ray diffraction were interpreted using computer software (Bruker AXS K.K.).

\subsection{MCZ Dissolution Test}

A FD was placed in a plastic dish and $10 \mathrm{~mL}$ of the dissolution medium (physiological saline preheated to $37^{\circ} \mathrm{C}$ ) was added. The dish was shaken at $300 \mathrm{rpm}$ in a shaker incubator (SI-300; As One Co., Osaka, Japan) at $37^{\circ} \mathrm{C}$. After 1, 3, 5, 10, 15, 20, 30, 45 and 60 minutes, $0.3 \mathrm{ml}$ of the medium was removed using a plastic syringe and filtered through a syringe driven filter unit (pore size: $0.45 \mu \mathrm{m})$. Then, $80-\mu \mathrm{l}$ aliquots of the filtered solution were placed into micro-test tubes $(1.5 \mathrm{ml})$ and $720 \mu \mathrm{l}$ of methanol was added to precipitate the polysaccharide dissolved from the dosage form. Samples were mixed and centrifuged $(7700 \times$ g, 5 min; H-1300; Kokusan Co., Saitama, Japan) and the supernatants were injected onto the HPLC column. All tests were performed in triplicate.

\section{Results and Discussion}

All polysaccharides used in this study formed films when the solvent was evaporated from the solution containing MCZ. As shown in Figure 1, 1.5\% ALG formed a circular 


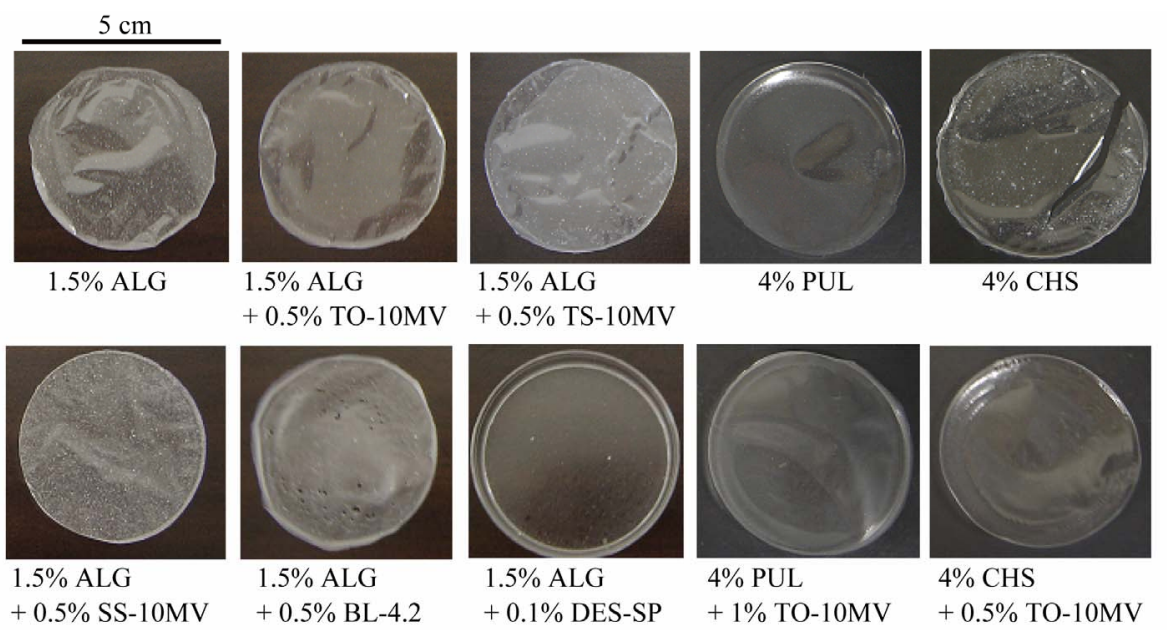

Figure 1. Images of FDs prepared with polysaccharides containing MCZ.

film (thickness, $74 \pm 4 \mu \mathrm{m}$ ) and MCZ was homogeneously dispersed in the film. Similar films were obtained from ALG solutions containing 0.5\% TO-10MV, TS-10MV or SS-10MV, with thicknesses of $70 \pm 3 \mu \mathrm{m}$, $84 \pm 7 \mu \mathrm{m}$ or $132 \pm 2 \mu \mathrm{m}$, respectively. Addition of a surfactant to the film base solution affected film formation. For example, pores were observed in the film prepared with $1.5 \%$ ALG containing $0.5 \%$ BL-4.2. The film prepared with $1.5 \%$ ALG containing $0.5 \%$ TL-10 was cracked, and $1.5 \%$ ALG containing 0.1\% DES-SP did not form a film. Circular films were obtained when $1.5 \%$ ALG-M, 4\% - 6\% PUL or $4 \%$ CHS was used as the base solution in the presence of $0.5 \%$ TO-10MV. The presence of $1 \%$ TO-10MV or $1 \%$ TS-10MV in the base solution of polysaccharide in the absence of PUL resulted in the formation of cracked films.

FDs containing MCZ are directly applied to the target region in the oral cavity; therefore, the form must be easy to handle. Despite the addition of surfactant, 4\% PUL formed a rigid film. In contrast, films prepared with $1.5 \%$ ALG and 1.5\% ALG-M were soft. Figure 2 shows the additive effect of surfactant on the rheological properties of FDs prepared with ALG. All films, except FD containing SS-10MV, were easy to handle and resistant to tearing. On the other hand, the film prepared with $4 \%$ CHS was unsuitable as a FD because it was too soft to handle.

In the casting method, MCZ was dispersed in the film base solution and the drug was trapped in the polysaccharide matrix. This procedure might affect the crystal structure of $\mathrm{MCZ}$ incorporated in the FD. Figure 3 shows X-ray diffractograms of FDs containing MCZ. Harrow patterns were provided by all FDs, and diffraction peaks arising from crystalline MCZ were not observed. These data show that MCZ may be incorporated in the amorphous form in the film matrix and that little crystalline form MCZ is present in the FDs.

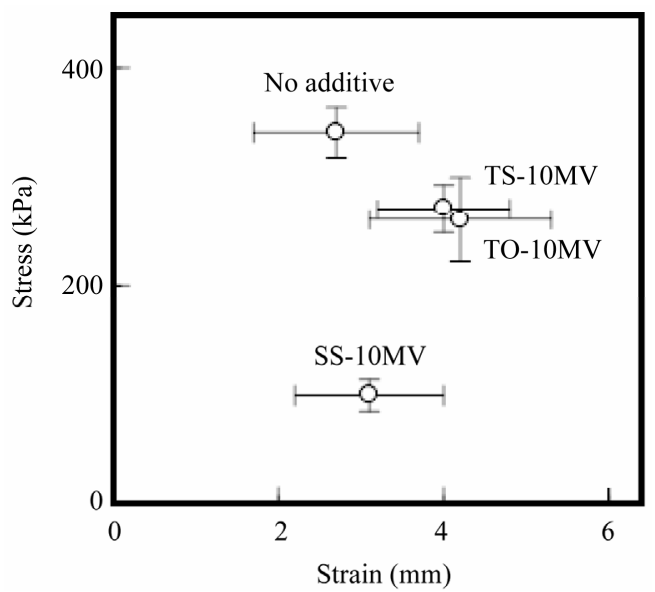

Figure 2. Rheological properties of FDs prepared with $1.5 \%$ ALG containing $0.5 \%$ surfactant. (No additive: surfactant free).

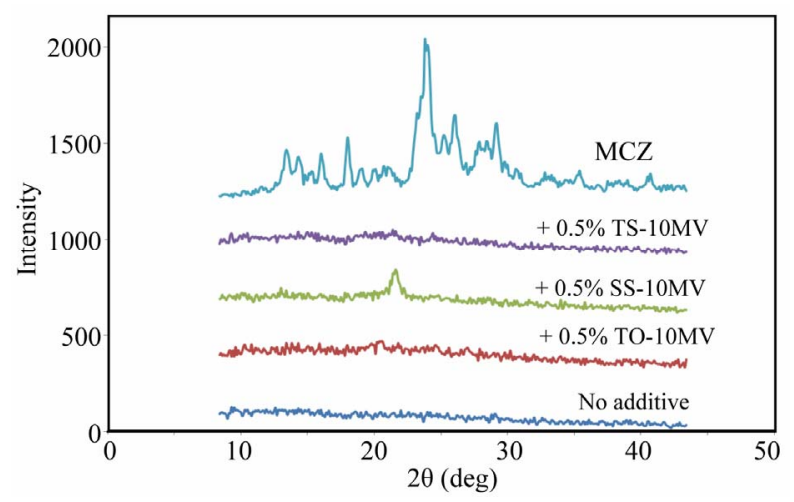

Figure 3. X-ray diffractograms of FDs prepared with $1.5 \%$ ALG containing MCZ. (No additive: surfactant free, MCZ: drug powder).

FDs prepared with water-soluble polysaccharides disintegrate when the form is dipped into aqueous medium. FD prepared with $1.5 \%$ ALG immediately swelled and 
disintegrated in $10 \mathrm{ml}$ of physiological saline at $37^{\circ} \mathrm{C}$. However, the concentration of MCZ in the medium did not increase quickly. Figure 4 shows the dissolution of MCZ from FD prepared with $1.5 \%$ ALG. MCZ gradually dissolved after the solid particles of drug were released from the FD. The MCZ concentration at 30 min was $49 \pm$ $2 \mu \mathrm{g} / \mathrm{ml}$, which is about $20 \%$ of the MCZ incorporated in the FD. The dissolution profile may be attributable to the poor solubility of the drug in physiological saline (saturated solubility, $0.49 \mathrm{mg} / \mathrm{ml}$ ). The solubility of MCZ was increased by the addition of surfactants utilized in this study, except for SS-10MV, as shown in Table 1. When FD prepared with $1.5 \%$ ALG was dipped into the test solution containing $0.15 \% \mathrm{TO}-10 \mathrm{MV}$ or TS-10MV, the MCZ dissolution rate increased, although about $50 \%$ of the drug added to the FD remained undissolved at 30 min.

As shown in Figure 5, the MCZ dissolution profile from FD was significantly altered by the incorporation of a surfactant such as TO-10MV into the form. As FD prepared with $1.5 \%$ ALG containing $0.5 \%$ TO-10MV started to dissolve into physiological saline, so too did MCZ. More than $50 \%$ of the MCZ incorporated into the FD

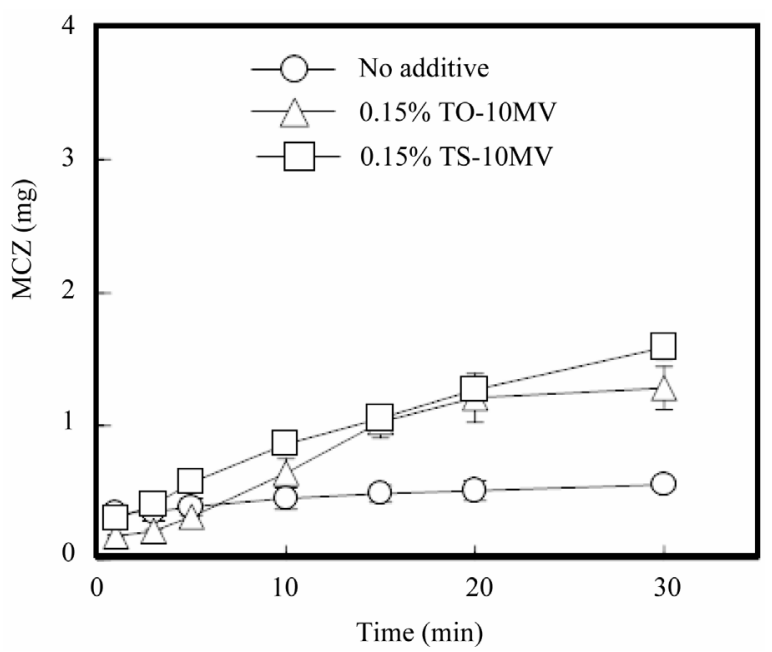

Figure 4. Release profiles of MCZ from FDs prepared with 1.5\% ALG in physiological saline containing a surfactant. (No additive: surfactant free).

Table 1. Solubility of MCZ in physiological saline containing a surfactant at $37^{\circ} \mathrm{C}$.

\begin{tabular}{lc}
\hline Surfactant & Solubility $(\mathrm{mg} / \mathrm{ml})$ \\
\hline No additives & 0.49 \\
$0.15 \%$ TO-10MV & 0.89 \\
$0.15 \%$ TS-10MV & 0.90 \\
$0.15 \%$ SS-10MV & 0.49 \\
$0.15 \%$ BL-4.2 & 1.25 \\
$0.15 \%$ DES-SP & $<0.49$ \\
\hline
\end{tabular}

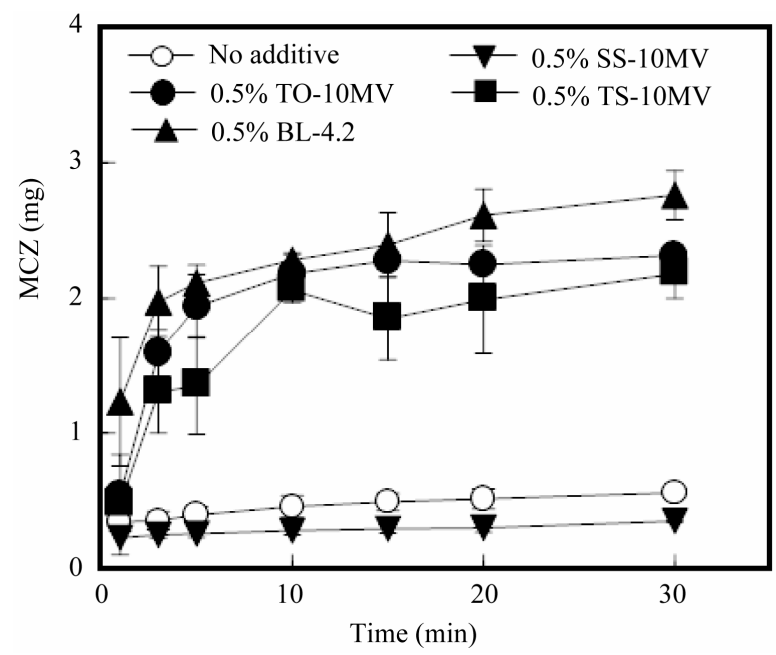

Figure 5. Release profiles of MCZ from FDs modified by the addition of a surfactant. (No additive: surfactant free).

was dissolved by $3 \mathrm{~min}$. A similar fast drug dissolution rate was observed in the case of FD containing TS-10MV or BL-4.2, but not when the FD contained SS-10MV. Figure 6 shows the additive effect of surfactant on the MCZ dissolution rate from FD. As the amount of TO$10 \mathrm{MV}$ or TS-10MV incorporated in the FD increased, the drug dissolution rate increased. A marked acceleration in the MCZ dissolution rate was also observed when the FD was prepared with other polysaccharides containing a surfactant. About $2 \mathrm{mg}$ of MCZ dissolved after 10 min when FD was prepared with either $1.5 \%$ ALG-M containing $0.5 \% \mathrm{TO}-10 \mathrm{MV}$ or $4 \%$ PUL containing $1 \%$ TO-10MV, as shown in Figure 7. These results indicate that the MCZ dissolution rate in an aqueous medium is accelerated by adding a surfactant to the base solution of the FD. This fast dissolution may be attributable to the coexistence of MCZ with a surfactant in the polysaccharide polymer matrix.

To treat oral candidiasis, MCZ is administered in the oral cavity to act directly at the affected site. It is probable that MCZ dissolves immediately upon contact with saliva, secreted at $1.5-2.0 \mathrm{ml} / \mathrm{min}$ from the salivary glands following stimulation [16]. MCZ must be applied repeatedly to completely treat the disease. In this study, FDs were prepared from safe materials, including surfactants such as TO-10MV. MCZ incorporated into such FDs can dissolve into a small amount of saliva as soon as the form disintegrates. Therefore, FDs modified with a surfactant are useful for treating localized problems in the oral cavity, such as oral candidiasis, and also simplify the administration of drugs to patients.

\section{Acknowledgements}

The authors would like to thank Dr. Hasebe (Industrial Research Institute of Ishikawa, Japan) for his help and 

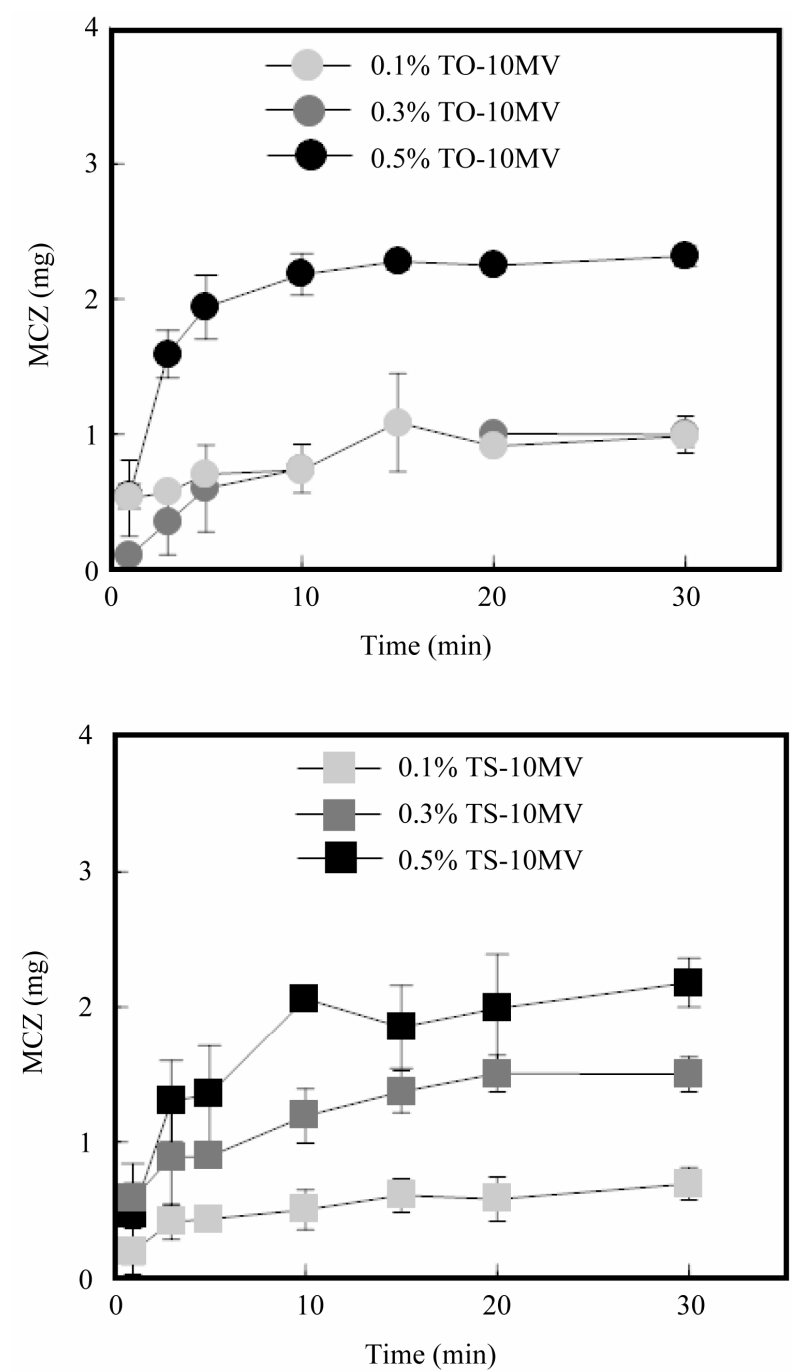

Figure 6. Effect of surfactant concentration on MCZ release from FDs.

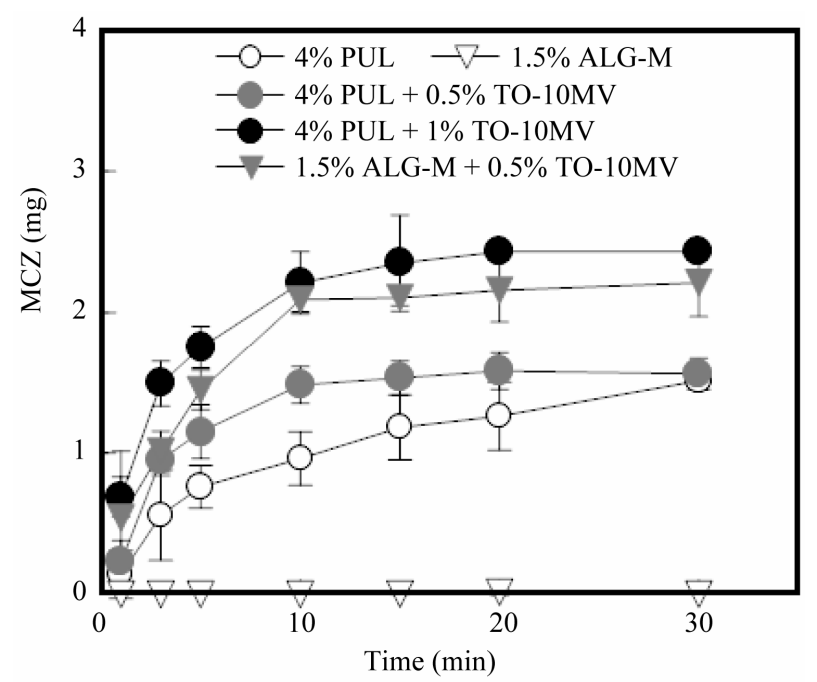

Figure 7. Release profiles of MCZ from FDs modified by the addition of a surfactant. advice with regard to X-ray diffractometry.

\section{REFERENCES}

[1] V. Garsuch and J. Breitkreutz, "Comparative Investigations on Different Polymers for the Preparation of FastDissolving Oral Films," Journal of Pharmacy and Pharmacology, Vol. 62, No. 4, 2010, pp. 539-545.

[2] T. Nagaraju, R. Gowthami, M. Rajashekar, S. Sandeep, M. Mallesham, D. Sathish and Y. S. Kumar, "Comprehensive Review on Oral Disintegrating Films," Current Drug Delivery, Vol. 10, No. 1, 2013, pp. 96-108. doi: $10.2174 / 1567201811310010016$

[3] K. B. Liew, Y. T. Tan and K. K. Peh, "Effect of Polymer, Plasticizer and Filler on Orally Disintegrating Film," Drug Development and Industrial Pharmacy, Posted Online, 2013.

[4] Y. Murata, T. Isobe, K. Kofuji, N. Nishida and R. Kamaguchi, "Preparation of Fast Dissolving Films for Oral Dosage from Natural Polysaccharides," Materials, Vol. 3, No. 8, 2010, pp. 4291-4299. doi:10.3390/ma3084291

[5] Y. Murata, K. Kofuji, N. Nishida and R. Kamaguchi, "Development of Film Dosage Form Containing Allopurinol for Prevention of Oral Mucositis," International Scholarly Research Network Pharmaceutics, 2012, 5 p, Article ID: 764510. doi:10.5402/2012/764510

[6] P. Siukosaari, S. Ajwani, A. Ainamo, J. Wolf and T. Närhi, "Periodontal Health Status in the Elderly with Different Levels of Education: A 5-Year Follow-Up Study," Gerodontology, Vol. 29, No. 2, 2012, pp. 170-178. doi:10.1111/j.1741-2358.2010.00437.x

[7] S. Offenbacher, S. P. Barros, S. Altarawneh, J. D. Beck and Z. G. Loewy, "Impact of Tooth Loss on Oral and Systemic Health," General Dentistry, Vol. 60, No. 6, 2012, pp. 494-500.

[8] P. A. Krishnan, "Fungal Infections of the Oral Mucosa," Indian Journal Dental Research, Vol. 23, No. 5, 2012, pp. 650-659. doi:10.4103/0970-9290.107384

[9] N. Isham and M. A. Ghannoum, "Antifungal Activity of Miconazole against Recent Candida Strains," Mycoses, Vol. 53, No. 5, 2010, pp. 434-437. doi:10.1111/j.1439-0507.2009.01728.x

[10] C. D. Collins, S. Cookinham and J. Smith, "Management of Oropharyngeal Candidiasis with Localized Oral Miconazole Therapy: Efficacy, Safety, and Patient Acceptability," Patient Preference and Adherence, Vol. 5, 2011, pp. 369-374. doi:10.2147/PPA.S14047

[11] J. Gronlund, T. I. Saari, N. Hagelberg, P. J. Neuvonen, K. T. Olkkola and K. Laine, "Miconazole Oral Gel Increases Exposure to Oral Oxycodone by Inhibition of CYP2D6 and CYP3A4," Antimicrobial Agents and Chemotherapy, Vol. 55, No. 3, 2011, pp. 1063-1067. doi:10.1128/AAC.01242-10

[12] R. J. Bensadoun, J. Daoud, B. E. Gueddari, L. Bastit, R. Gourmet, A. Rosikon, C. Allavena, P. Ceruse, G. Calais and P. Attali, "Comparison of the Efficacy and Safety of Miconazole $50 \mathrm{mg}$ Mucoadhesive Buccal Tablets with Miconazole $500 \mathrm{mg} \mathrm{Gel}$ in the Treatment of Oropharyn- 
geal Candidiasis," Cancer, Vol. 112, No. 1, 2008, pp. 204211. doi: $10.1002 /$ cncr.23152

[13] A. Madgulkar, S. Kadam and V. Pokharkar, "Development of Buccal Adhesive Tablet with Prolonged Antifungal Activity: Optimization and ex Vivo Deposition Studies," Indian Journal Pharmaceutical Sciences, Vol. 71, No. 3, 2009, pp. 290-294.

[14] M. R. Bhalekar, V. Pokharkar, A. Madgulkar, N. Patil and N. Patil, "Preparation and Evaluation of Miconazole Nitrate-Loaded Solid Liquid Nanoparticles for Topical Delivery," American Association of Pharmaceutical Sci-

\section{Abbreviations}

ALG: sodium alginate

ALG-M: mannuronic acid-rich ALG

BL-4.2: polyoxyethylene lauryl ether

CHS: chondroitin sulfate C-type

DES-SP: diethyl sebacate

FD: film dosage form entists PharmSciTech, Vol. 10, No. 1, 2009, pp. 289-296.

[15] L. K. Pershing, J. Corlett and C. Jorgensen, "In Vivo Pharmacokinetics and Pharmacodynamics of Topical Ketoconazole and Miconazole in Human Stratum Corneum," Antimicrobial Agents and Chemotherapy, Vol. 38, No. 1, 1994, pp. 90-95. doi:10.1128/AAC.38.1.90

[16] B. Nauntofte, J. O. Tenovuo and F. Lagerlof, "Secretion and Composition of Saliva," In: O. Fejerskov and E. A. M. Kidd, Eds., Dental Caries the Disease and Its Clinical Management, Blackwell Munksgaad, Oxford, 2003, pp. 7-27.

MCZ: miconazole

PUL: pullulan

SS-10MV: sorbitan stearate

TL-10: polyoxyethylene sorbitan fatty acid ester

TO-10MV: polyoxyethylene sorbitan monooleate

TS-10MV: polyoxyethylene sorbitan monostearate 